\title{
Effect of Long-Term Use of Inorganic Fertilizers, Organic Manures and their Combination on Soil Properties and Enzyme Activity in Rice-Rice Cropping System
}

\author{
N. Goutami*, Ch. Sujani Rao, A. Sireesha, Ch. Pulla Rao and A. Vijaya Gopal \\ Department of Soil Science and Agricultural Chemistry, Agricultural College, \\ Bapatla-522 101, Andhra Pradesh, India \\ *Corresponding author
}

A B S T R A C T

\begin{tabular}{|c|c|}
\hline & \multirow{7}{*}{$\begin{array}{l}\text { A field experiment entitled "Carbon sequestration and soil health under long term soil } \\
\text { fertility management in rice-rice cropping system" was carried out under field conditions } \\
\text { during kharif and rabi seasons of } 2016-2017 \text { and } 2017-2018 \text { at Andhra Pradesh Rice } \\
\text { Research Institute and Regional Agricultural Research Station, Maruteru, West Godavari } \\
\text { district in the ongoing All India Coordinated Research Project on Long Term Fertilizer } \\
\text { Experiment Project. The results indicated that at initial, tillering, panicle initiation and at } \\
\text { harvest stage, significantly the highest available nitrogen, phosphorus, potassium and } \\
\text { micronutrients in soil were recorded with application of } 100 \% \mathrm{NPK}+\mathrm{ZnSO}_{4}+\mathrm{FYM}\left(\mathrm{T}_{7}\right) \text {. } \\
\text { The treatments } \mathrm{T}_{9}\left(50 \% \mathrm{NPK}+50 \% \mathrm{~N} \text { through green manures), } \mathrm{T}_{10}(50 \% \mathrm{NPK}+50 \%\right. \\
\mathrm{N} \text { through FYM }) \text { and } \mathrm{T}_{11}(50 \% \mathrm{NPK}+25 \% \mathrm{~N} \text { through } \mathrm{FYM}+25 \% \mathrm{~N} \text { through green } \\
\text { manures) were on par with each other in all four seasons of study. The highest enzyme } \\
\text { activity was observed with application of } 100 \% \text { NPK }+\mathrm{ZnSO}_{4}+\mathrm{FYM}\left(\mathrm{T}_{7}\right) \text { (both Kharif } \\
\text { and Rabi) which was significantly superior over remaining treatments in (Kharif, } 2016 \text { and } \\
\text { Rabi, 2017). The application of zinc did not show any significant effect on enzyme } \\
\text { activity. }\end{array}$} \\
\hline & \\
\hline Organic $\mathrm{m}$ & \\
\hline $\begin{array}{l}\text { Inorganics, Urease, } \\
\text { Available nutrients }\end{array}$ & \\
\hline Article Info & \\
\hline $\begin{array}{l}\text { Accepted: } \\
\text { 06 August } 2018 \\
\text { Available Online: } \\
\text { 10 September } 2018\end{array}$ & \\
\hline & \\
\hline
\end{tabular}

\section{Introduction}

Rice-rice, the main cropping system in the eastern coast of India, requires heavy amount of plant nutrients that results in decline in net returns per unit area (Anonymous, 2001). Soil fertility and productivity in Godavari delta are likely to be affected due to intensive rice monoculture with imbalanced fertilization under excessive use of irrigation water. A declining trend in the productivity of rice even when grown under adequate application of $\mathrm{N}$,
$\mathrm{P}$ and $\mathrm{K}$ was reported by Nambiar and Abrol (1989). Continuous use of high level of chemical fertilizers had lead to soil degradation problems, which also proved detrimental to soil health.

This paper is a part of the first author's Ph.D. (Ag.) thesis entitled "Carbon sequestration and soil health under long term soil fertility management in rice-rice cropping system", submitted to Acharya N.G. Ranga Agricultural University, Lam, Guntur. 
Many tropical soils are poor in nutrients and rely on the recycling of nutrients from soil organic matter to improve and maintain crop productivity. Intensive cultivation, growing of exhaustive crops, use of imbalanced and inadequate fertilizers, restricted use of organic manures has made the soils not only deficient in nutrients but also deteriorate soil health resulting decline in crop response to recommended dose of NPK fertilizers. To supply recommended dose of nutrients, large quantities of organic material is needed and also slow release of plant nutrients upon decomposition from organic material deprive crop growth. Under such conditions integrated plant nutrient management assumes greater significance and plays a vital role in maintenance of soil health and sustainable productivity.

\section{Materials and Methods}

The experiment was carried out under field conditions during kharif and rabi seasons of 2016-2017 and 2017- 2018 at Andhra Pradesh Rice Research Institute and Regional Agricultural Research Station, Maruteru, West Godavari district in the ongoing All India Coordinated Research Project on Long Term Fertilizer Experiment Project. The treatments consisted of control, 100 per cent recommended dose of NPK, 100 per cent recommended dose of NK, 100 per cent recommended dose of $\mathrm{PK}, 100$ per cent recommended dose of NP, 100 per cent recommended dose of $\mathrm{NPK}+\mathrm{ZnSO}_{4}$ @ $40 \mathrm{~kg}$ / ha, 100 per cent recommended dose of $\mathrm{NPK}+\mathrm{ZnSO}_{4} @ 40 \mathrm{~kg} / \mathrm{ha}+\mathrm{FYM} @ 5 \mathrm{t} \mathrm{ha}^{-1}$, 50 per cent recommended dose of NPK, $50 \%$ $\mathrm{NPK}+50 \% \mathrm{~N}$ through green manures, $50 \%$ $\mathrm{NPK}+50 \% \mathrm{~N}$ through FYM, $50 \% \mathrm{NPK}+$ $25 \% \mathrm{~N}$ through green manures $+25 \% \mathrm{~N}$ through FYM and FYM only @ 10 t/ha. All together there were twelve treatments laidout in RBD with three replications for both kharif and rabi seasons in two years of study.
Nitrogen was applied through urea in three equal splits $\left(1 / 3^{\text {rd }}\right.$ basal $+1 / 3^{\text {rd }}$ at tillering $+1 / 3^{\text {rd }}$ at panicle initiation stage). Phosphorus was applied through DAP was used duly taking its $\mathrm{N}$ content into account and potassium as muriate of potash $\left(60 \% \mathrm{~K}_{2} \mathrm{O}\right)$ and zinc as zinc sulphate $\left(\mathrm{ZnSO}_{4} .7 \mathrm{H}_{2} \mathrm{O}\right)$. The entire dose of phosphorus, potassium and zinc were applied as basal. Recommended dose of fertilizer for kharif season was 90: 60: $60 \mathrm{~N}: \mathrm{P}_{2} \mathrm{O}_{5}: \mathrm{K}_{2} \mathrm{O} \mathrm{kg}$ $\mathrm{ha}^{-1}$ and for rabi season it was 180: 90: $60 \mathrm{~N}$ : $\mathrm{P}_{2} \mathrm{O}_{5}: \quad \mathrm{K}_{2} \mathrm{O} \quad \mathrm{kg} \mathrm{ha}^{-1}$. Well decomposed farmyard manure (FYM) manure and Calotropis (green leaf manure) were applied two weeks before transplanting. The experiment on rice - rice sequence as detailed above was repeated on a same site during kharif 2016-17 and rabi 2017-18, respectively. Popular cultivars of kharif rice and rabi rice, MTU-1061, MTU-1010 respectively, were used for the study. Data was collected on available nutrients and enzyme activities of both kharif and rabi rice.

\section{Results and Discussion}

\section{Nutrient status of soil}

Available nutrient status of soil was significantly influenced by long-term use of different organics, inorganics and their combination during both the years of the study.

\section{Nitrogen}

At initial stage, the highest available nitrogen in soil was recorded in treatment $\mathrm{T}_{7}(100 \%$ $\mathrm{RDF}+\mathrm{ZnSO}_{4}+\mathrm{FYM}$ ) and it was significantly superior over all other treatments during kharif and rabi in two years of study and the lowest available nitrogen was observed in control $\left(\mathrm{T}_{1}\right)$. Similar trends were observed at initial stage of rabi rice with $279.6,243.8 \mathrm{~kg} \mathrm{ha}^{-1}$, respectively. The available nitrogen values were higher during rabi due to residual effect 
of applied nutrients to kharif rice and also due to application of relatively higher doses of inorganic fertilizers to rabi rice. Among the inorganic treatments $\left(\mathrm{T}_{2}, \mathrm{~T}_{3}, \mathrm{~T}_{4}, \mathrm{~T}_{5}, \mathrm{~T}_{6}\right.$ and $\mathrm{T}_{8}$ ), the treatment $\mathrm{T}_{6}$ was recorded highest available nitrogen and significantly superior over $\mathrm{T}_{4}, \mathrm{~T}_{5}$ and $\mathrm{T}_{8}$ but however it was on par with $T_{2}, T_{3}$. Similar results were obtained in both the years of study during kharif and rabi season at initial stage. These results were in close conformity with the findings of Kumar and Singh (2010).

The organic treatments, $T_{9}, T_{10}$ and $T_{11}$ were on par with each other. The results were in consonance with the findings of Malewer and Hasnabade (1995) who reported significant increase in available nitrogen with application of organics and inorganics and sustained the productivity. The minimum available nitrogen was observed in control $\left(\mathrm{T}_{1}\right)$. At initial, tillering, panicle initiation and at harvesting stage, significantly the highest $(239.2,274.3$, $258.6,241.5$ and $243.1,278.5,257.8,245.3 \mathrm{~kg}$ $\mathrm{ha}^{-1}$, in kharif, 2016 and 17 available nitrogen in soil was recorded respectively with application of $100 \% \mathrm{NPK}+\mathrm{ZnSO}_{4}+\mathrm{FYM}$ $\left(\mathrm{T}_{7}\right)$. Similar trend was observed in rabi season with 279.6, 240.9, 260.4, 244.3 and 243.8, 276.9, 259.8, 249.8 kg ha ${ }^{-1}$ in $\mathrm{T}_{7}$ in Rabi 2017 and 18, respectively.

With respect to tillering stage the highest available nitrogen was observed in $\mathrm{T}_{7}(100 \%$ $\mathrm{NPK}+\mathrm{ZnSO} 4+$ FYM) and it was significantly superior over other treatments and the lowest soil available nitrogen was observed in control treatment $\left(\mathrm{T}_{1}\right)$. Similar set of results were observed in kharif and rabi during both the years of study. Among the inorganic treatments the treatment $\mathrm{T}_{6}$ was recorded highest available nitrogen and significantly superior over $\mathrm{T}_{3}, \mathrm{~T}_{4}, \mathrm{~T}_{5}$ and $\mathrm{T}_{8}$ and however it was on par with treatment $\mathrm{T}_{2}$. Similar results were obtained in both the years of study in kharif and rabi.
At panicle initiation stage, significantly higher value of the available nitrogen was observed in $\mathrm{T}_{7}\left(100 \% \mathrm{NPK}+\mathrm{ZnSO}_{4}+\mathrm{FYM}\right)$ which was significantly superior over all other treatments and the lowest available nitrogen was observed in $\mathrm{T}_{1}$ (absolute control). Similar results were obtained in both the years of study in kharif and rabi. Among the inorganic treatments $\left(\mathrm{T}_{2}, \mathrm{~T}_{3}, \mathrm{~T}_{4}, \mathrm{~T}_{5}, \mathrm{~T}_{6}\right.$ and $\left.\mathrm{T}_{8}\right)$, the treatment $\mathrm{T}_{6}$ was recorded highest available nitrogen and significantly superior over $\mathrm{T}_{4}$ and $\mathrm{T}_{8}$ but however it was on par with $\mathrm{T}_{2}, \mathrm{~T}_{3}$ and $\mathrm{T}_{5}$. Similar results were obtained in both the years of study in kharif and rabi.

At harvest stage, in kharif, rabi 2016-17 and 2017-18 the highest available nitrogen was observed in $\mathrm{T}_{7}(50 \%$ RDP + FYM) which was significantly superior over all other treatments and lowest was observed in control treatment $\left(\mathrm{T}_{1}\right)$. Among inorganic treatments the treatment $\mathrm{T}_{6}\left(100 \% \mathrm{RDF}+\mathrm{ZnSO}_{4}\right)$ was recorded highest available nitrogen and it was significantly superior over all other inorganic treatments. This increase in available nitrogen content can be attributed to the mineralisation of soil nitrogen leading to build up of available nitrogen (Swarup and Yaduvanshi, 2000).

Irrespective of treatments, the available nitrogen in soil was higher at initial stages of crop and declined to the later stages. This might be due to the uptake of $\mathrm{N}$ by the growing plants as reported by Prakash and Badrinath (1994) (Table 1 and 2).

\section{Phosphorus}

At initial stage, the higher available phosphorus (61.2 and $65.3 \mathrm{~kg} \mathrm{P}_{2} \mathrm{O}_{5}$ ha $^{-1}$ during 2016 and 2017, respectively) in kharif, whereas in rabi the higher available phosphorus (65.9 and $64.1 \mathrm{~kg} \mathrm{P}_{2} \mathrm{O}_{5} \mathrm{ha}^{-1}$ during 2017 and 2018, respectively) was recorded with the treatment of $100 \% \mathrm{RDF}+\mathrm{ZnSO} 4+$ 
FYM $\left(\mathrm{T}_{7}\right)$ it was significantly superior over other treatments but however it was on par with treatment $\left(\mathrm{T}_{2}\right)$ 100\% RDF (59.4, 59.8; $60.8,59.4 \mathrm{~kg} \mathrm{P}_{2} \mathrm{O}_{5} \mathrm{ha}^{-1}$ ) during kharif and rabi in 2016-17 and 2017-18, respectively) and lowest phosphorus $(35.8,28.1 ; 31.9,26.1 \mathrm{~kg}$ $\mathrm{P}_{2} \mathrm{O}_{5} \mathrm{ha}^{-1}$ ) was observed in control.

At tillering stage, among different treatments, the treatment $\mathrm{T}_{7}$ recorded highest available $\mathrm{P}_{2} \mathrm{O}_{5} 71.3,78.4 ; 75.8,74.8 \mathrm{~kg} \mathrm{ha}^{-1}$ in kharif, rabi 2016-17 and kharif rabi 2017-18 and it was significantly superior over other treatments but however it was on par with treatment $\mathrm{T}_{2}\left(67.5,73.9 ; 70.9,74.8 \mathrm{~kg} \mathrm{ha}^{-1}\right.$ in kharif, rabi 2016-17 and kharif rabi 2017-18) and lowest available phosphorus was observed in control. The enhanced availability of soil $\mathrm{P}$ at tillering of rice might be due to the production of organic acids during microbial decomposition of the crop residues in soil.

The significant increase in $\mathrm{P}$ status of soil in these treatments could be due to the fact that organic acids released during decomposition of FYM might have converted the unavailable forms of phosphorus to available forms besides mineralization of organic form of phosphorus. These results confirm the findings of Lakshminarayana (2006).

At panicle initiation stage, the highest value of the available phosphorus was observed in $\mathrm{T}_{7}$ $\left(100 \% \quad \mathrm{NPK}+\mathrm{ZnSO}_{4}+\mathrm{FYM}\right)$ it was significantly superior over all other treatments. The lowest available nitrogen was observed in $\mathrm{T}_{1}$ (absolute control). The increase in available phosphorus status of soil from sowing to maximum tillering stage can be attributed to the flooding condition of the soil and it was in conformity with the results of Chakravarthi and Kar (1970).

The treatment $\mathrm{T}_{7}(100 \% \mathrm{RDF}+\mathrm{FYM}+$ $\mathrm{ZnSO}_{4}$ ) recorded significantly highest (64.4, 66.3; 66.8, $69.3 \mathrm{Kg} \mathrm{P}_{2} \mathrm{O}_{5} \mathrm{ha}^{-1}$ in kharif, rabi
2016-17 and kharif rabi 2017-18) available phosphorus at harvest stage during both kharif and rabi. Lowest $(32.4,26.5$ and $28.9,25.1 \mathrm{~kg}$ $\mathrm{ha}^{-1}$ in kharif, rabi 2016-17 and kharif rabi 2017-18respectively) available phosphorus was recorded with $\mathrm{T}_{1}$ (absolute control). The higher available phosphorus in farmyard manure amended treatments might be due to the release of $\mathrm{P}$ from the well decomposed FYM.

Among the inorganic treatments $\left(\mathrm{T}_{2}, \mathrm{~T}_{3}, \mathrm{~T}_{4}, \mathrm{~T}_{5}\right.$ $\mathrm{T}_{6}$ and $\mathrm{T}_{8}$ ), the treatment $\mathrm{T}_{2}$ recorded the highest available phosphorus and it was significantly superior over $\mathrm{T}_{3}$ and $\mathrm{T}_{8}$. However it was on par with $\mathrm{T}_{4}, \mathrm{~T}_{5}$ and $\mathrm{T}_{6}$. The treatment $\mathrm{T}_{2}(100 \% \mathrm{NPK})$ was on par with $\mathrm{T}_{6}$ $\left(100 \% \mathrm{NPK}+\mathrm{ZnSO}_{4}\right)$ at all stages of crop growth during both the years of study in kharif and rabi season (Table 3 and 4).

\section{Potassium (kg ha $\left.{ }^{-1}\right)$}

Available potassium in soils at initial stage ranged from 304.5 to 383.9 ; 299.1 to 384.1 ; and 302.1 to $385.9 ; 297.1$ to $385.3 \mathrm{~kg} \mathrm{~K}_{2} \mathrm{O}$ $\mathrm{ha}^{-1}$, in kharif and rabi during 2016-17 and 2017-18, respectively during the two years of study. At initial stage the highest available $\mathrm{K}_{2} \mathrm{O}$ content was observed in $\mathrm{T}_{7}$ treatment that received 100\% RD of NPK+FYM @ $5 \mathrm{t} \mathrm{ha}^{-1}$ which was significantly superior over other treatments but however it was on par with treatment $\mathrm{T}_{6}\left(100 \% \mathrm{NPK}+\mathrm{ZnSO}_{4}\right)$ in both kharif seasons and rabi, 2017 whereas in rabi 2018, the treatment $T_{7}$ was significantly superior over all other treatments and the lowest available potassium was observed in control $\left(\mathrm{T}_{1}\right)$ and this his was on par with $\mathrm{T}_{5}$ (100\% RD of NP) and $\mathrm{T}_{8}(50 \% \mathrm{NPK})$.

At tillering stage, available potassium in soils was ranged from 302.4 to $426.8 ; 298.3$ to 429.3 ; and 301.4 to $435.9 ; 296.3$ to $437.9 \mathrm{~kg}$ $\mathrm{K}_{2} \mathrm{O}$ ha $^{-1}$, in kharif, rabi 2016-17 and 2017-18 respectively. The highest available potassium 
was observed in $\mathrm{T}_{7}(100 \% \mathrm{RD}$ of $\mathrm{NPK}+\mathrm{FYM}$ @ $5 \mathrm{t} \mathrm{ha}^{-1}+\mathrm{ZnSO}_{4}$ ) and it was significantly superior over other treatments but however it was on par with $\mathrm{T}_{6}$ and $\mathrm{T}_{2}$ in Rabi season. Whereas in kharif season the treatment $\mathrm{T}_{7}$ was significantly superior over other treatments but however it was on par with $\mathrm{T}_{6}$. Obviously control $\left(\mathrm{T}_{1}\right)$ showed comparatively the lowest available potassium content.

Among the nutrient management treatments, application of NPK + FYM to rice recorded higher quantity of available soil NPK after crop harvest. This might be due to slow release of nutrients in FYM and also due to the chelating effect of FYM. Rathore et al., (1995) also observed that residual soil fertility increased under FYM application, whereas, NPK alone made no impact on fertility buildup.

The beneficial effect of FYM on available potassium might be due to the reduction of potassium fixation, solubilisation and release due to the interaction of organic matter with clay besides the direct potassium addition to the potassium pool of soil. Similar results were observed by Tandon, 1987.

Among the treatments, higher (395.4, 394.5 and $399.5,404.2 \mathrm{~kg} \mathrm{~K} \mathrm{~K}_{2} \mathrm{O} \mathrm{ha}^{-1}$ ) values of available $\mathrm{K}$ content were recorded at panicle initiation stage with application of $100 \%$ $\mathrm{NPK}+\mathrm{FYM}+\mathrm{ZnSO}_{4}$ which was significantly superior over remaining treatments in kharif season during both the years of study. Whereas in rabi the treatment $\mathrm{T}_{7}$ was significantly superior over other treatments but however it was on par with treatment $\mathrm{T}_{6}$ and lower available potassium content was recorded in control treatment $\left(\mathrm{T}_{1}\right)$.

At harvest, the highest available potassium (385.6, 386.9 and $386.7,391.5 \mathrm{~kg} \mathrm{~K}_{2} \mathrm{O} \mathrm{ha}{ }^{-1}$ ) was observed in $\mathrm{T}_{7}$ which was significantly superior over remaining treatments in both kharif and rabi seasons and the lowest (301.5, 297.3 and 299.8, $295.8 \mathrm{~kg} \mathrm{~K}_{2} \mathrm{O} \mathrm{ha}^{-1}$ ) available potassium was observed in control, which was on par with $\mathrm{T}_{5}(100 \% \mathrm{NP})$.

Among the inorganic treatments $\left(\mathrm{T}_{2}, \mathrm{~T}_{3}, \mathrm{~T}_{4}\right.$, $\mathrm{T}_{5}, \mathrm{~T}_{6}$ and $\mathrm{T}_{8}$ ), the highest available potassium was observed in treatment $\mathrm{T}_{6}$ it was significantly superior over $\mathrm{T}_{5}(100 \% \mathrm{NP})$ and $\mathrm{T}_{8} \quad\left(\begin{array}{lll}50 & \% & \mathrm{NPK}\end{array}\right)$ and however it was statistically on par with $\mathrm{T}_{2}(100 \% \mathrm{RDF}), \mathrm{T}_{3}$ $(100 \% \mathrm{NK}), \mathrm{T}_{4}(100 \% \mathrm{PK})$. Similar results were obtained at all stages of crop growth during both the years of study in kharif and rabi season (Table 5 and 6).

\section{Enzyme activities}

\section{Urease activity}

The data relating the effect of different treatments on activity of soil urease ( $\mu \mathrm{g}$ of $\mathrm{NH} 4^{+}$released $\mathrm{g}^{-1}$ soil $\mathrm{h}^{-1}$ ) at various growth stages of rice are presented in Table 7 and 8 . A close perusal of data indicated significant differences between treatments at all the crop growth stages. In all the treatments, the urease activity showed an increasing trend with the age of the crop and exhibited highest activity at panicle initiation stage and thereafter the activity decreased towards harvest. From the tillering to filling stages, the rice roots excreted more organic acids and carbohydrates, which stimulated the correlative soil enzymatic activities. The results corroborate with the findings of Zeng et al., (2005). During kharif, 2016 and 2017, significantly high urease activity of 42.34 , 46.84, 52.19, 44.59 and 46.82, 51.29, 55.49, $48.38 \mathrm{mg} \mathrm{NH}_{4}^{+}$released $\mathrm{g}^{-1}$ soil $\mathrm{h}^{-1}$ was recorded by $\mathrm{T}_{7}\left(100 \% \mathrm{NPK}+\mathrm{FYM}+\mathrm{ZnSO}_{4}\right)$ followed by $\mathrm{T}_{10}(50 \% \mathrm{NPK}+50 \% \mathrm{~N}$ through FYM with 40.04, 43.61, 48.39, 41.64 and 43.21, 48.93, 52.81, $44.69 \mathrm{mg} \mathrm{NH}_{4}^{+}$released $\mathrm{g}^{-1}$ soil $\mathrm{h}^{-1}$ at initial, tillering, panicle initiation and at harvest was recorded, respectively. 
Table.1 Effect of long-term use of inorganic fertilizers, organic manures and their combination on soil available nitrogen $\left(\mathrm{kg}^{-1}\right)$

\begin{tabular}{|c|c|c|c|c|c|c|c|c|}
\hline \multirow[t]{2}{*}{ Treatments } & \multicolumn{4}{|c|}{ Kharif (2016) } & \multicolumn{4}{|c|}{ Rabi (2017) } \\
\hline & Initial & Tillering & $\begin{array}{l}\text { Panicle } \\
\text { Initiation }\end{array}$ & Harvest & Initial & Tillering & $\begin{array}{l}\text { Panicle } \\
\text { Initiation }\end{array}$ & Harvest \\
\hline $\mathbf{T}_{1}$ Control & 161.8 & 160.4 & 159.3 & 158.6 & 156.4 & 155.3 & 153.8 & 152.1 \\
\hline $\mathrm{T}_{2} 100 \% \mathrm{RDF}$ & 222.9 & 256.3 & 240.8 & 226.5 & 224.9 & 265.9 & 239.1 & 225.9 \\
\hline $\mathrm{T}_{3} 100 \% \mathrm{NK}$ & 216.3 & 254.9 & 232.4 & 216.7 & 214.7 & 263.1 & 233.8 & 221.4 \\
\hline $\mathrm{T}_{4} 100 \% \mathrm{PK}$ & 176.4 & 212.7 & 186.7 & 178.3 & 175.3 & 225.8 & 193.3 & 177.3 \\
\hline $\mathrm{T}_{5} \mathbf{1 0 0 \%} \mathrm{NP}$ & 215.6 & 251.8 & 231.6 & 215.9 & 214.9 & 261.7 & 230.8 & 216.8 \\
\hline $\mathrm{T}_{6} 100 \% \mathrm{RDF}+\mathrm{ZnSO}_{4} @ 40 \mathrm{~kg} / \mathrm{ha}$ & 224.3 & 260.5 & 243.5 & 230.4 & 227.4 & 267.3 & 243.5 & 228.4 \\
\hline $\mathrm{T}_{7} 100 \% \mathrm{RDF}+\mathrm{ZnSO}_{4} @ 40 \mathrm{~kg} / \mathrm{ha}+\mathrm{FYM} @ 5 \mathrm{t} / \mathrm{ha}$ & 239.2 & 274.3 & 258.6 & 241.5 & 240.9 & 279.6 & 260.4 & 244.3 \\
\hline $\mathrm{T}_{8} \mathbf{5 0 \%} \mathrm{NPK}$ & 190.4 & 229.9 & 202.5 & 196.6 & 190.8 & 239.5 & 209.2 & 193.6 \\
\hline $\mathrm{T}_{9} \mathbf{5 0 \%} \mathrm{NPK}+\mathbf{5 0} \% \mathrm{~N}$ Through Green Manures & 212.3 & 252.3 & 235.1 & 218.8 & 215.3 & 257.8 & 235.1 & 218.7 \\
\hline $\mathrm{T}_{10} \mathbf{5 0 \%} \mathrm{NPK}+50 \% \mathrm{~N}$ Through FYM & 213.6 & 253.5 & 238.6 & 219.4 & 217.4 & 260.4 & 236.8 & 220.6 \\
\hline $\begin{array}{l}\mathrm{T}_{11} 50 \% \mathrm{NPK}+25 \% \mathrm{~N} \text { Through GM + } 25 \% \mathrm{~N} \\
\text { Through FYM }\end{array}$ & 210.6 & 250.7 & 234.4 & 217.9 & 214.6 & 259.3 & 231.4 & 219.5 \\
\hline T12FYM only@10 t/ha & 195.4 & 238.5 & 212.5 & 199.4 & 196.8 & 245.5 & 213.8 & 198.8 \\
\hline $\mathrm{SEm} \pm$ & 4.603 & 4.364 & 4.944 & 4.194 & 5.080 & 3.853 & 5.387 & 5.592 \\
\hline CD@0.05 & 13.5 & 12.8 & 14.5 & 12.3 & 14.9 & 11.3 & 15.8 & 16.4 \\
\hline CV (\%) & 11.16 & 10.68 & 10.52 & 9.82 & 9.57 & 8.46 & 10.68 & 9.15 \\
\hline
\end{tabular}


Table.2 Effect of long-term use of inorganic fertilizers, organic manures and their combination on soil available nitrogen $\left(\mathrm{kg}^{-1} \mathrm{a}^{-1}\right)$

\begin{tabular}{|c|c|c|c|c|c|c|c|c|}
\hline \multirow[t]{2}{*}{ Treatments } & \multicolumn{4}{|c|}{ Kharif (2017) } & \multicolumn{4}{|c|}{ Rabi (2018) } \\
\hline & Initial & Tillering & $\begin{array}{l}\text { Panicle } \\
\text { Initiation }\end{array}$ & Harvest & Initial & Tillering & $\begin{array}{l}\text { Panicle } \\
\text { Initiation }\end{array}$ & Harvest \\
\hline $\mathbf{T}_{1}$ Control & 151.2 & 149.6 & 148.3 & 147.1 & 146.3 & 145.9 & 145.1 & 144.3 \\
\hline $\mathrm{T}_{2} 100 \% \mathrm{RDF}$ & 224.3 & 262.9 & 245.6 & 224.6 & 223.6 & 264.7 & 242.3 & 230.4 \\
\hline $\mathrm{T}_{3} 100 \% \mathrm{NK}$ & 219.4 & 254.4 & 236.5 & 217.8 & 215.9 & 258.1 & 234.4 & 225.9 \\
\hline $\mathrm{T}_{4} \mathbf{1 0 0 \%} \mathrm{PK}$ & 173.8 & 220.1 & 208.4 & 176.4 & 174.5 & 226.8 & 196.7 & 180.3 \\
\hline $\mathrm{T}_{5} 100 \% \mathrm{NP}$ & 215.1 & 252.7 & 237.1 & 215.3 & 213.8 & 253.1 & 235.9 & 221.5 \\
\hline $\mathrm{T}_{6} 100 \% \mathrm{RDF}+\mathrm{ZnSO}_{4} @ 40 \mathrm{~kg} / \mathrm{ha}$ & 224.8 & 264.3 & 246.3 & 230.8 & 229.7 & 268.3 & 243.3 & 233.5 \\
\hline $\mathrm{T}_{7} 100 \% \mathrm{RDF}+\mathrm{ZnSO}_{4} @ 40 \mathrm{~kg} / \mathrm{ha}+\mathrm{FYM} @ 5 \mathrm{t} / \mathrm{ha}$ & 243.1 & 278.5 & 257.8 & 245.3 & 243.8 & 276.9 & 259.8 & 249.8 \\
\hline $\mathrm{T}_{8} \mathbf{5 0 \%} \mathrm{NPK}$ & 190.4 & 229.8 & 219.3 & 191.5 & 189.6 & 230.7 & 208.8 & 197.8 \\
\hline $\mathrm{T}_{\mathbf{9}} \mathbf{5 0 \%} \mathrm{NPK}+\mathbf{5 0} \% \mathrm{~N}$ Through Green Manures & 216.5 & 251.5 & 238.6 & 220.6 & 218.3 & 253.8 & 231.6 & 222.5 \\
\hline $\mathrm{T}_{10} \mathbf{5 0 \%} \mathrm{NPK}+\mathbf{5 0} \% \mathrm{~N}$ Through FYM & 218.3 & 253.6 & 240.9 & 221.3 & 220.5 & 259.7 & 239.4 & 228.9 \\
\hline $\begin{array}{l}\mathrm{T}_{11} 50 \% \mathrm{NPK}+25 \% \mathrm{~N} \text { Through GM + } 25 \% \mathrm{~N} \\
\text { Through FYM }\end{array}$ & 217.6 & 250.9 & 237.8 & 219.5 & 218.9 & 255.6 & 235.6 & 224.8 \\
\hline T12FYM only@10t/ha & 196.3 & 234.6 & 225.2 & 198.4 & 197.9 & 239.6 & 216.5 & 203.6 \\
\hline $\mathrm{SEm} \pm$ & 5.148 & 4.739 & 3.512 & 4.057 & 3.716 & 5.043 & 4.732 & 4.362 \\
\hline CD@0.05 & 15.1 & 13.9 & 10.3 & 11.9 & 10.9 & 14.8 & 13.9 & 12.8 \\
\hline $\mathrm{CV}(\%)$ & 9.65 & 8.93 & 7.16 & 10.95 & 7.83 & 8.16 & 7.92 & 8.36 \\
\hline
\end{tabular}


Table.3 Effect of long-term use of inorganic fertilizers, organic manures and their combination on soil available phosphorus $\left(\mathrm{kg} \mathrm{ha}^{-1}\right)$

\begin{tabular}{|c|c|c|c|c|c|c|c|c|}
\hline \multirow[t]{2}{*}{ Treatments } & \multicolumn{4}{|c|}{ Kharif (2016) } & \multicolumn{4}{|c|}{ Rabi (2017) } \\
\hline & Initial & Tillering & $\begin{array}{l}\text { Panicle } \\
\text { Initiation }\end{array}$ & Harvest & Initial & Tillering & $\begin{array}{l}\text { Panicle } \\
\text { Initiation }\end{array}$ & Harvest \\
\hline $\mathrm{T}_{1}$ Control & 35.8 & 34.9 & 33.3 & 32.4 & 31.9 & 30.8 & 29.5 & 28.9 \\
\hline $\mathrm{T}_{2} 100 \% \mathrm{RDF}$ & 59.4 & 67.5 & 61.3 & 60.3 & 59.4 & 70.9 & 63.4 & 60.9 \\
\hline $\mathrm{T}_{3} \mathbf{1 0 0 \%} \mathrm{NK}$ & 36.1 & 44.4 & 42.8 & 37.3 & 41.2 & 49.4 & 43.5 & 42.1 \\
\hline $\mathrm{T}_{4} 100 \% \mathrm{PK}$ & 53.2 & 64.3 & 55.6 & 54.9 & 55.1 & 67.8 & 58.9 & 56.3 \\
\hline $\mathrm{T}_{5} \mathbf{1 0 0 \%} \mathrm{NP}$ & 53.8 & 63.8 & 54.7 & 54.1 & 54.3 & 68.1 & 58.1 & 55.8 \\
\hline $\mathrm{T}_{6} 100 \% \mathrm{RDF}+\mathrm{ZnSO}_{4} @ 40 \mathrm{~kg} / \mathrm{ha}$ & 56.0 & 65.2 & 59.6 & 57.3 & 56.2 & 69.3 & 62.9 & 57.8 \\
\hline $\mathrm{T}_{7} 100 \% \mathrm{RDF}+\mathrm{ZnSO}_{4} @ 40 \mathrm{~kg} / \mathrm{ha}+\mathrm{FYM} @ 5 \mathrm{t} / \mathrm{ha}$ & 61.2 & 71.3 & 65.7 & 64.4 & 64.1 & 75.8 & 69.7 & 66.8 \\
\hline $\mathrm{T}_{8} \mathbf{5 0 \%} \mathrm{NPK}$ & 48.6 & 52.4 & 50.1 & 49.3 & 48.2 & 55.4 & 49.4 & 48.9 \\
\hline $\mathrm{T}_{9} \mathbf{5 0 \%} \mathrm{NPK}+\mathbf{5 0} \% \mathrm{~N}$ Through Green Manures & 54.1 & 59.8 & 56.3 & 56.2 & 55.8 & 67.3 & 60.2 & 57.4 \\
\hline $\mathrm{T}_{10} \mathbf{5 0 \%} \mathrm{NPK}+\mathbf{5 0} \% \mathrm{~N}$ Through FYM & 55.5 & 62.8 & 58.3 & 57.3 & 56.1 & 66.8 & 61.8 & 58.9 \\
\hline $\begin{array}{l}\mathrm{T}_{11} 50 \% \mathrm{NPK}+25 \% \mathrm{~N} \text { Through GM + } 25 \% \mathrm{~N} \\
\text { Through FYM }\end{array}$ & 54.3 & 61.4 & 55.6 & 55.4 & 53.4 & 65.1 & 59.3 & 55.3 \\
\hline T12 FYM only@10 t/ha & 49.1 & 55.2 & 50.4 & 47.5 & 49.1 & 59.3 & 51.1 & 50.1 \\
\hline $\mathrm{SEm} \pm$ & 1.193 & 2.012 & 2.319 & 1.364 & 1.671 & 1.739 & 2.080 & 2.012 \\
\hline CD@0.05 & 3.5 & 5.9 & 6.8 & 4.0 & 4.9 & 5.1 & 6.1 & 5.9 \\
\hline CV $(\%)$ & 10.6 & 8.1 & 8.3 & 9.5 & 9.5 & 9.0 & 8.9 & 11.2 \\
\hline
\end{tabular}


Table.4 Effect of long-term use of inorganic fertilizers, organic manures and their combination on available phosphorus $\left(\mathrm{kg}^{-1}\right)$

\begin{tabular}{|c|c|c|c|c|c|c|c|c|}
\hline \multirow{2}{*}{ Treatments } & \multicolumn{4}{|c|}{ Kharif (2017) } & \multicolumn{4}{|c|}{ Rabi (2018) } \\
\hline & Initial & Tillering & $\begin{array}{l}\text { Panicle } \\
\text { Initiation }\end{array}$ & Harvest & Initial & Tillering & $\begin{array}{l}\text { Panicle } \\
\text { Initiation }\end{array}$ & Harvest \\
\hline $\mathrm{T}_{1}$ Control & 28.1 & 27.5 & 27.0 & 26.5 & 26.1 & 26.0 & 25.8 & 25.1 \\
\hline $\mathrm{T}_{2} 100 \% \mathrm{RDF}$ & 59.8 & 73.9 & 65.8 & 61.9 & 60.8 & 74.8 & 66.4 & 62.5 \\
\hline $\mathrm{T}_{3} \mathbf{1 0 0 \%} \mathrm{NK}$ & 41.4 & 53.4 & 48.5 & 43.5 & 42.8 & 57.6 & 52.9 & 47.8 \\
\hline $\mathrm{T}_{4} 100 \% \mathrm{PK}$ & 55.9 & 68.1 & 58.6 & 56.3 & 55.3 & 70.5 & 63.4 & 58.1 \\
\hline $\mathrm{T}_{5} 100 \% \mathrm{NP}$ & 54.1 & 69.3 & 59.3 & 55.4 & 54.1 & 69.3 & 64.7 & 57.1 \\
\hline $\mathrm{T}_{6} 100 \% \mathrm{RDF}+\mathrm{ZnSO}_{4} @ 40 \mathrm{~kg} / \mathrm{ha}$ & 57.5 & 70.5 & 63.5 & 59.6 & 58.9 & 73.4 & 65.3 & 61.3 \\
\hline $\mathrm{T}_{7} 100 \% \mathrm{RDF}+\mathrm{ZnSO}_{4} @ 40 \mathrm{~kg} / \mathrm{ha}+\mathrm{FYM} @ 5 \mathrm{t} / \mathrm{ha}$ & 65.3 & 78.4 & 70.3 & 66.3 & 65.9 & 80.3 & 74.5 & 69.3 \\
\hline $\mathrm{T}_{8} \mathbf{5 0 \%} \mathrm{NPK}$ & 48.7 & 58.5 & 52.9 & 49.4 & 48.3 & 61.7 & 57.6 & 52.9 \\
\hline T, $50 \%$ NPK + $50 \%$ N Through Green Manures & 56.5 & 67.1 & 61.5 & 58.3 & 57.6 & 70.3 & 65.1 & 58.9 \\
\hline $\mathrm{T}_{10} \mathbf{5 0 \%} \mathrm{NPK}+\mathbf{5 0} \% \mathrm{~N}$ Through FYM & 57.3 & 67.4 & 62.8 & 59.1 & 58.5 & 71.9 & 66.9 & 59.8 \\
\hline $\begin{array}{l}\mathrm{T}_{11} 50 \% \mathrm{NPK}+25 \% \mathrm{~N} \text { Through GM }+25 \% \mathrm{~N} \\
\text { Through FYM }\end{array}$ & 54.9 & 66.3 & 60.1 & 55.3 & 54.4 & 69.7 & 64.3 & 58.7 \\
\hline T12 FYM only@10t/ha & 49.5 & 61.0 & 54.5 & 50.9 & 50.1 & 63.1 & 58.2 & 54.2 \\
\hline SEm \pm & 1.807 & 1.637 & 1.330 & 1.432 & 1.534 & 1.296 & 1.398 & 1.603 \\
\hline CD@ 0.05 & 5.3 & 4.8 & 3.9 & 4.2 & 4.5 & 3.8 & 4.1 & 4.7 \\
\hline CV (\%) & 8.8 & 10.9 & 8.9 & 9.5 & 9.1 & 7.6 & 7.3 & 8.3 \\
\hline
\end{tabular}


Table.5 Effect of long-term use of inorganic fertilizers, organic manures and their combination on available potassium $\left(\mathrm{kg} \mathrm{ha}^{-1}\right)$

\begin{tabular}{|c|c|c|c|c|c|c|c|c|}
\hline \multirow[t]{2}{*}{ Treatments } & \multicolumn{4}{|c|}{ Kharif (2016) } & \multicolumn{4}{|c|}{ Rabi (2017) } \\
\hline & Initial & Tillering & $\begin{array}{l}\text { Panicle } \\
\text { Initiation }\end{array}$ & Harvest & Initial & Tillering & $\begin{array}{l}\text { Panicle } \\
\text { Initiation }\end{array}$ & Harvest \\
\hline $\mathrm{T}_{1}$ Control & 304.5 & 302.4 & 301.8 & 301.5 & 302.1 & 301.4 & 300.8 & 299.8 \\
\hline $\mathrm{T}_{2} 100 \% \mathrm{RDF}$ & 363.5 & 405.5 & 372.6 & 364.3 & 364.9 & 417.8 & 380.5 & 365.4 \\
\hline $\mathrm{T}_{3} \mathbf{1 0 0 \% \mathrm { NK }}$ & 349.6 & 403.8 & 361.8 & 350.8 & 350.1 & 411.7 & 373.1 & 351.8 \\
\hline $\mathrm{T}_{4} 100 \% \mathrm{PK}$ & 347.1 & 401.6 & 365.4 & 349.7 & 348.6 & 409.3 & 370.2 & 350.2 \\
\hline $\mathrm{T}_{5} \mathbf{1 0 0 \% \mathrm { NP }}$ & 306.4 & 337.2 & 321.8 & 308.6 & 308.1 & 350.6 & 325.8 & 309.5 \\
\hline $\mathrm{T}_{6} 100 \% \mathrm{RDF}+\mathrm{ZnSO}_{4} @ 40 \mathrm{~kg} / \mathrm{ha}$ & 365.2 & 411.3 & 375.5 & 366.4 & 365.6 & 419.3 & 382.4 & 366.9 \\
\hline $\mathrm{T}_{7} 100 \% \mathrm{RDF}+\mathrm{ZnSO}_{4} @ 40 \mathrm{~kg} / \mathrm{ha}+\mathrm{FYM} @ 5 \mathrm{t} / \mathrm{ha}$ & 383.9 & 426.8 & 395.4 & 385.6 & 385.9 & 435.9 & 399.5 & 386.7 \\
\hline $\mathrm{T}_{8} \mathbf{5 0 \%} \mathrm{NPK}$ & 318.6 & 362.1 & 330.8 & 320.4 & 319.5 & 375.5 & 344.3 & 320.6 \\
\hline $\mathrm{T}_{9} \mathbf{5 0 \%} \mathrm{NPK}+\mathbf{5 0} \% \mathrm{~N}$ Through Green Manures & 356.4 & 397.6 & 367.7 & 358.6 & 357.8 & 403.1 & 375.7 & 359.1 \\
\hline $\mathrm{T}_{10} \mathbf{5 0 \%} \mathrm{NPK}+\mathbf{5 0} \% \mathrm{~N}$ Through FYM & 358.2 & 399.7 & 370.1 & 359.5 & 358.9 & 405.6 & 378.9 & 360.2 \\
\hline $\begin{array}{l}\mathrm{T}_{11} 50 \% \mathrm{NPK}+25 \% \mathrm{~N} \text { Through GM }+25 \% \mathrm{~N} \\
\text { Through FYM }\end{array}$ & 354.9 & 395.4 & 368.2 & 356.4 & 356.1 & 401.2 & 376.4 & 357.3 \\
\hline T12 FYM only@10t/ha & 326.2 & 374.8 & 342.9 & 328.1 & 327.2 & 382.7 & 352.6 & 329.1 \\
\hline SEm \pm & 6.444 & 6.751 & 6.581 & 5.967 & 7.228 & 6.205 & 5.830 & 6.478 \\
\hline CD@0.05 & 18.9 & 19.8 & 19.3 & 17.5 & 21.2 & 18.2 & 17.1 & 19.0 \\
\hline CV (\%) & 7.9 & 8.5 & 10.1 & 6.7 & 8.3 & 7.1 & 10.5 & 9.8 \\
\hline
\end{tabular}


Table.6 Effect of long-term use of inorganic fertilizers, organic manures and their combination on available potassium $\left(\mathrm{kg} \mathrm{ha}^{-1}\right)$

\begin{tabular}{|c|c|c|c|c|c|c|c|c|}
\hline \multirow[t]{2}{*}{ Treatments } & \multicolumn{4}{|c|}{ Kharif (2017) } & \multicolumn{4}{|c|}{ Rabi (2018) } \\
\hline & Initial & Tillering & $\begin{array}{l}\text { Panicle } \\
\text { Initiation }\end{array}$ & Harvest & Initial & Tillering & $\begin{array}{l}\text { Panicle } \\
\text { Initiation }\end{array}$ & Harvest \\
\hline$T_{1}$ Control & 299.1 & 298.3 & 298.1 & 297.3 & 297.1 & 296.3 & 296.1 & 295.8 \\
\hline $\mathrm{T}_{2} 100 \% \mathrm{RDF}$ & 364.2 & 409.6 & 375.8 & 366.5 & 365.8 & 420.5 & 388.4 & 369.1 \\
\hline $\mathrm{T}_{3} 100 \% \mathrm{NK}$ & 350.6 & 408.2 & 368.4 & 353.4 & 352.7 & 403.1 & 373.9 & 354.5 \\
\hline $\mathrm{T}_{4} 100 \% \mathrm{PK}$ & 349.1 & 406.1 & 367.5 & 351.6 & 351.1 & 401.9 & 372.6 & 352.3 \\
\hline $\mathrm{T}_{5} \mathbf{1 0 0 \% \mathrm { NP }}$ & 310.7 & 349.8 & 320.4 & 313.7 & 312.2 & 363.8 & 333.2 & 309.5 \\
\hline $\mathrm{T}_{6} 100 \% \mathrm{RDF}+\mathrm{ZnSO}_{4} @ 40 \mathrm{~kg} / \mathrm{ha}$ & 365.2 & 410.1 & 377.5 & 367.4 & 366.2 & 421.9 & 390.9 & 372.4 \\
\hline $\mathrm{T}_{7} 100 \% \mathrm{RDF}+\mathrm{ZnSO}_{4} @ 40 \mathrm{~kg} / \mathrm{ha}+\mathrm{FYM} @ 5 \mathrm{t} / \mathrm{ha}$ & 384.1 & 429.3 & 394.5 & 386.9 & 385.3 & 437.9 & 404.2 & 391.5 \\
\hline $\mathrm{T}_{8} \mathbf{5 0 \%} \mathrm{NPK}$ & 317.9 & 368.6 & 343.9 & 319.8 & 319.1 & 370.2 & 339.7 & 323.2 \\
\hline $\mathrm{T}_{9} \mathbf{5 0 \%} \mathrm{NPK}+\mathbf{5 0} \% \mathrm{~N}$ Through Green Manures & 357.3 & 401.5 & 370.8 & 359.1 & 358.9 & 412.7 & 380.2 & 362.5 \\
\hline $\mathrm{T}_{10} 50 \% \mathrm{NPK}+50 \% \mathrm{~N}$ Through FYM & 360.1 & 403.8 & 373.6 & 363.8 & 362.7 & 417.9 & 384.3 & 364.9 \\
\hline $\begin{array}{l}\mathrm{T}_{11} 50 \% \mathrm{NPK}+25 \% \mathrm{~N} \text { Through GM + } 25 \% \mathrm{~N} \\
\text { Through FYM }\end{array}$ & 356.8 & 398.6 & 369.7 & 359.6 & 357.3 & 413.7 & 382.7 & 361.7 \\
\hline T12 FYM only@10 t/ha & 329.0 & 375.7 & 348.6 & 331.2 & 330.8 & 383.9 & 366.2 & 332.4 \\
\hline $\mathrm{SEm} \pm$ & 6.751 & 6.308 & 5.558 & 6.103 & 5.524 & 5.967 & 6.171 & 6.069 \\
\hline CD@0.05 & 19.8 & 18.5 & 16.3 & 17.9 & 16.2 & 17.5 & 18.1 & 17.8 \\
\hline CV (\%) & 9.1 & 7.5 & 8.9 & 10.6 & 9.5 & 8.2 & 7.7 & 8.5 \\
\hline
\end{tabular}


Table.7 Effect of long-term use of inorganic fertilizers, organic manures and their combination on soil urease activity $\left(\mu \mathrm{g} \mathrm{NH}{ }_{4}^{+}-\mathrm{N} \mathrm{g}^{-1}\right.$ soil h$\left.^{-1}\right)$

\begin{tabular}{|c|c|c|c|c|c|c|c|c|}
\hline \multirow[t]{2}{*}{ Treatments } & \multicolumn{4}{|c|}{ Kharif (2016) } & \multicolumn{4}{|c|}{ Rabi (2017) } \\
\hline & Initial & Tillering & $\begin{array}{l}\text { Panicle } \\
\text { Initiation }\end{array}$ & Harvest & Initial & Tillering & $\begin{array}{l}\text { Panicle } \\
\text { Initiation }\end{array}$ & Harvest \\
\hline $\mathrm{T}_{1}$ Control & 21.34 & 26.39 & 28.43 & 24.16 & 22.14 & 27.94 & 31.27 & 25.38 \\
\hline $\mathrm{T}_{2} 100 \% \mathrm{RDF}$ & 30.64 & 35.16 & 38.18 & 33.34 & 32.09 & 38.63 & 43.96 & 37.17 \\
\hline $\mathrm{T}_{3} 100 \% \mathrm{NK}$ & 28.39 & 34.39 & 33.81 & 31.98 & 30.18 & 35.68 & 41.08 & 34.31 \\
\hline $\mathrm{T}_{4} \mathbf{1 0 0 \%} \mathrm{PK}$ & 26.16 & 31.38 & 31.68 & 28.16 & 27.62 & 33.18 & 37.79 & 33.69 \\
\hline $\mathrm{T}_{5} \mathbf{1 0 0 \%} \mathrm{NP}$ & 27.19 & 32.49 & 34.79 & 31.14 & 29.94 & 36.19 & 40.29 & 35.72 \\
\hline $\mathrm{T}_{6} 100 \% \mathrm{RDF}+\mathrm{ZnSO}_{4} @ 40 \mathrm{~kg} / \mathrm{ha}$ & 31.96 & 36.24 & 39.38 & 34.18 & 33.41 & 39.19 & 45.24 & 38.84 \\
\hline $\mathrm{T}_{7} 100 \% \mathrm{RDF}+\mathrm{ZnSO}_{4} @ 40 \mathrm{~kg} / \mathrm{ha}+\mathrm{FYM} @ 5 \mathrm{t} / \mathrm{ha}$ & 42.34 & 46.84 & 52.19 & 44.59 & 43.69 & 50.34 & 58.94 & 48.13 \\
\hline $\mathrm{T}_{8} \mathbf{5 0 \%} \mathrm{NPK}$ & 25.39 & 29.14 & 30.18 & 26.93 & 26.32 & 32.79 & 36.18 & 31.19 \\
\hline T, 50\% NPK + $50 \%$ N Through Green Manures & 37.84 & 42.64 & 46.31 & 39.59 & 38.14 & 44.18 & 52.31 & 43.26 \\
\hline $\mathrm{T}_{10} \mathbf{5 0 \%} \mathrm{NPK}+\mathbf{5 0} \% \mathrm{~N}$ Through FYM & 40.04 & 43.61 & 48.39 & 41.64 & 40.16 & 47.28 & 55.01 & 44.19 \\
\hline $\begin{array}{l}\mathrm{T}_{11} 50 \% \mathrm{NPK}+25 \% \mathrm{~N} \text { Through GM + } 25 \% \mathrm{~N} \\
\text { Through FYM }\end{array}$ & 36.39 & 40.38 & 44.56 & 39.32 & 39.15 & 45.39 & 51.38 & 45.63 \\
\hline T12 FYM only@10 t/ha & 34.16 & 38.38 & 41.93 & 37.50 & 36.19 & 42.24 & 48.14 & 41.34 \\
\hline $\mathrm{SEm} \pm$ & 0.839 & 1.139 & 1.238 & 0.736 & 1.292 & 1.371 & 1.320 & 0.999 \\
\hline CD@0.05 & 2.46 & 3.34 & 3.63 & 2.16 & 3.79 & 4.02 & 3.87 & 2.93 \\
\hline $\mathrm{CV}(\%)$ & 8.19 & 7.68 & 8.23 & 9.16 & 7.04 & 7.47 & 8.39 & 7.89 \\
\hline
\end{tabular}


Table.8 Effect of long-term use of inorganic fertilizers, organic manures and their combination on soil urease activity $\left(\mu \mathrm{g} \mathrm{NH}_{4}{ }^{+}-\mathrm{N} \mathrm{g}^{-1}\right.$ soil h$\left.^{-1}\right)$

\begin{tabular}{|c|c|c|c|c|c|c|c|c|}
\hline \multirow[t]{2}{*}{ Treatments } & \multicolumn{4}{|c|}{ Kharif (2017) } & \multicolumn{4}{|c|}{ Rabi (2018) } \\
\hline & Initial & Tillering & $\begin{array}{l}\text { Panicle } \\
\text { Initiation }\end{array}$ & Harvest & Initial & Tillering & $\begin{array}{l}\text { Panicle } \\
\text { Initiation }\end{array}$ & Harvest \\
\hline $\mathrm{T}_{1}$ Control & 23.19 & 28.08 & 32.79 & 25.73 & 22.15 & 27.83 & 32.15 & 26.89 \\
\hline $\mathrm{T}_{2} \mathbf{1 0 0} \% \mathrm{RDF}$ & 35.24 & 39.13 & 42.81 & 36.89 & 34.89 & 39.14 & 44.81 & 38.24 \\
\hline $\mathrm{T}_{3} 100 \% \mathrm{NK}$ & 32.18 & 35.19 & 39.18 & 33.16 & 30.29 & 35.84 & 41.83 & 33.14 \\
\hline $\mathrm{T}_{4} \mathbf{1 0 0 \%} \mathrm{PK}$ & 31.16 & 34.96 & 37.49 & 32.17 & 29.14 & 32.16 & 35.92 & 31.84 \\
\hline $\mathrm{T}_{5} \mathbf{1 0 0 \%} \mathrm{NP}$ & 34.14 & 36.19 & 38.32 & 35.86 & 31.63 & 33.89 & 37.84 & 32.94 \\
\hline $\mathrm{T}_{6} 100 \% \mathrm{RDF}+\mathrm{ZnSO}_{4} @ 40 \mathrm{~kg} / \mathrm{ha}$ & 36.29 & 40.94 & 44.02 & 37.62 & 35.24 & 39.18 & 44.89 & 37.84 \\
\hline $\mathrm{T}_{7} 100 \% \mathrm{RDF}+\mathrm{ZnSO}_{4} @ 40 \mathrm{~kg} / \mathrm{ha}+\mathrm{FYM} @ 5 \mathrm{t} / \mathrm{ha}$ & 46.82 & 51.29 & 55.49 & 48.38 & 45.84 & 53.89 & 60.18 & 51.98 \\
\hline $\mathrm{T}_{8} \mathbf{5 0 \%} \mathrm{NPK}$ & 29.28 & 32.68 & 36.84 & 30.42 & 27.32 & 33.14 & 35.82 & 30.15 \\
\hline $\mathrm{T}_{9} \mathbf{5 0} \% \mathrm{NPK}+\mathbf{5 0} \% \mathrm{~N}$ Through Green Manures & 42.19 & 45.62 & 49.55 & 42.35 & 39.19 & 49.18 & 55.97 & 46.89 \\
\hline $\mathrm{T}_{10} 50 \% \mathrm{NPK}+50 \% \mathrm{~N}$ Through FYM & 42.36 & 48.93 & 52.81 & 44.69 & 41.52 & 51.62 & 59.73 & 49.18 \\
\hline $\begin{array}{l}\mathrm{T}_{11} 50 \% \mathrm{NPK}+25 \% \mathrm{~N} \text { Through GM }+25 \% \mathrm{~N} \\
\text { Through FYM }\end{array}$ & 43.21 & 46.37 & 51.13 & 43.28 & 40.13 & 48.34 & 52.63 & 45.92 \\
\hline T12 FYM only @10 t/ha & 39.36 & 42.73 & 45.86 & 39.07 & 35.24 & 45.85 & 51.13 & 43.81 \\
\hline SEm \pm & 1.333 & 1.190 & 1.418 & 1.197 & 1.146 & 1.377 & 1.016 & 1.279 \\
\hline CD@0.05 & 3.91 & 3.49 & 4.16 & 3.49 & 3.36 & 4.04 & 2.98 & 3.75 \\
\hline CV $(\%)$ & 7.26 & 7.91 & 7.73 & 8.48 & 7.16 & 8.28 & 7.97 & 7.34 \\
\hline
\end{tabular}


Table.9 Effect of long-term use of inorganic fertilizers, organic manures and their combination on soil dehydrogenase activity $\left(\mu \mathrm{g}\right.$ of TPF $\mathrm{g}^{-1}$ soil Day $^{-1}$ )

\begin{tabular}{|c|c|c|c|c|c|c|c|c|}
\hline \multirow[t]{2}{*}{ Treatments } & \multicolumn{4}{|c|}{ Kharif (2016) } & \multicolumn{4}{|c|}{ Rabi (2017) } \\
\hline & Initial & Tillering & $\begin{array}{l}\text { Panicle } \\
\text { Initiation }\end{array}$ & Harvest & Initial & Tillering & $\begin{array}{l}\text { Panicle } \\
\text { Initiation }\end{array}$ & Harvest \\
\hline $\mathrm{T}_{1}$ Control & 259.62 & 263.14 & 269.19 & 261.83 & 260.14 & 265.19 & 269.96 & 263.16 \\
\hline $\mathrm{T}_{2} 100 \% \mathrm{RDF}$ & 325.63 & 338.82 & 350.26 & 334.92 & 331.39 & 341.29 & 355.92 & 339.62 \\
\hline $\mathrm{T}_{3} 100 \% \mathrm{NK}$ & 295.28 & 326.93 & 339.17 & 319.62 & 317.82 & 329.62 & 343.19 & 323.32 \\
\hline $\mathrm{T}_{4} 100 \% \mathrm{PK}$ & 281.39 & 300.62 & 312.15 & 292.81 & 291.49 & 305.81 & 318.82 & 301.83 \\
\hline $\mathrm{T}_{5} 100 \% \mathrm{NP}$ & 300.86 & 312.39 & 321.29 & 309.65 & 307.89 & 317.89 & 327.76 & 312.96 \\
\hline $\mathrm{T}_{6} 100 \% \mathrm{RDF}+\mathrm{ZnSO}_{4} @ 40 \mathrm{~kg} / \mathrm{ha}$ & 331.92 & 347.21 & 354.01 & 340.38 & 338.12 & 350.13 & 361.32 & 345.82 \\
\hline $\mathrm{T}_{7} 100 \% \mathrm{RDF}+\mathrm{ZnSO}_{4} @ 40$ kg/ha + FYM @ 5t/ha & 416.31 & 424.29 & 439.36 & 421.38 & 420.16 & 429.19 & 446.89 & 426.23 \\
\hline $\mathrm{T}_{8} \mathbf{5 0 \%} \mathrm{NPK}$ & 262.93 & 274.89 & 281.59 & 269.89 & 267.83 & 281.18 & 286.17 & 279.14 \\
\hline $\mathrm{T}_{9} \mathbf{5 0 \%} \mathrm{NPK}+\mathbf{5 0} \% \mathrm{~N}$ Through Green Manures & 344.32 & 359.84 & 379.70 & 351.42 & 349.34 & 365.13 & 382.19 & 360.39 \\
\hline $\mathrm{T}_{10} \mathbf{5 0 \%} \mathrm{NPK}+\mathbf{5 0} \% \mathrm{~N}$ Through FYM & 375.66 & 388.01 & 399.13 & 383.29 & 380.62 & 388.96 & 409.13 & 383.42 \\
\hline $\begin{array}{l}\mathrm{T}_{11} 50 \% \mathrm{NPK}+25 \% \mathrm{~N} \text { Through GM + } 25 \% \mathrm{~N} \\
\text { Through FYM }\end{array}$ & 360.84 & 371.32 & 381.29 & 367.89 & 365.81 & 379.13 & 391.32 & 370.34 \\
\hline T12 FYM only@10 t/ha & 385.62 & 394.42 & 413.29 & 391.82 & 389.34 & 396.81 & 419.19 & 391.35 \\
\hline SEm \pm & 12.21 & 13.61 & 11.87 & 13.77 & 13.06 & 14.62 & 12.22 & 12.66 \\
\hline CD@ 0.05 & 35.81 & 39.92 & 34.84 & 40.39 & 38.32 & 42.89 & 35.86 & 37.13 \\
\hline CV (\%) & 7.89 & 8.13 & 8.64 & 9.13 & 7.32 & 8.16 & 7.52 & 8.35 \\
\hline
\end{tabular}


Table.10 Effect of long-term use of inorganic fertilizers, organic manures and their combination on soil dehydrogenase activity $\left(\mu \mathrm{g}\right.$ of TPF $\mathrm{g}^{-1}$ soil Day $^{-1}$ )

\begin{tabular}{|c|c|c|c|c|c|c|c|c|}
\hline \multirow[t]{2}{*}{ Treatments } & \multicolumn{4}{|c|}{ Kharif (2017) } & \multicolumn{4}{|c|}{ Rabi (2018) } \\
\hline & Initial & Tillering & $\begin{array}{l}\text { Panicle } \\
\text { Initiation }\end{array}$ & Harvest & Initial & Tillering & $\begin{array}{l}\text { Panicle } \\
\text { Initiation }\end{array}$ & Harvest \\
\hline $\mathrm{T}_{1}$ Control & 262.27 & 265.19 & 267.82 & 263.29 & 262.91 & 270.12 & 273.12 & 267.14 \\
\hline $\mathrm{T}_{2} 100 \% \mathrm{RDF}$ & 338.89 & 348.34 & 350.13 & 343.13 & 339.14 & 345.84 & 349.16 & 340.16 \\
\hline $\mathrm{T}_{3} 100 \% \mathrm{NK}$ & 321.16 & 330.19 & 339.72 & 327.21 & 323.81 & 330.28 & 335.82 & 306.28 \\
\hline $\mathrm{T}_{4} 100 \% \mathrm{PK}$ & 300.82 & 309.62 & 315.84 & 305.32 & 292.87 & 299.84 & 304.12 & 293.62 \\
\hline $\mathrm{T}_{5} \mathbf{1 0 0 \%} \mathrm{NP}$ & 311.16 & 319.34 & 326.82 & 315.82 & 313.86 & 318.69 & 324.89 & 315.81 \\
\hline $\mathrm{T}_{6} 100 \% \mathrm{RDF}+\mathrm{ZnSO}_{4} @ 40 \mathrm{~kg} / \mathrm{ha}$ & 342.19 & 352.16 & 359.74 & 349.39 & 343.81 & 346.82 & 353.28 & 345.72 \\
\hline $\mathrm{T}_{7} 100 \% \mathrm{RDF}+\mathrm{ZnSO}_{4} @ 40 \mathrm{~kg} / \mathrm{ha}+\mathrm{FYM} @ 5 \mathrm{t} / \mathrm{ha}$ & 425.21 & 434.91 & 449.82 & 430.32 & 427.69 & 435.62 & 446.81 & 431.84 \\
\hline $\mathrm{T}_{8} \mathbf{5 0 \%} \mathrm{NPK}$ & 278.29 & 283.19 & 296.81 & 281.26 & 274.83 & 279.89 & 283.29 & 276.15 \\
\hline T, $50 \%$ NPK + $50 \%$ N Through Green Manures & 359.13 & 369.82 & 379.95 & 364.19 & 359.19 & 365.39 & 370.82 & 361.06 \\
\hline $\mathrm{T}_{10} 50 \% \mathrm{NPK}+50 \% \mathrm{~N}$ Through FYM & 381.42 & 393.65 & 404.13 & 389.27 & 385.24 & 391.41 & 396.79 & 389.96 \\
\hline $\begin{array}{l}\mathrm{T}_{11} 50 \% \mathrm{NPK}+25 \% \mathrm{~N} \text { Through GM + } 25 \% \mathrm{~N} \\
\text { Through FYM }\end{array}$ & 368.19 & 379.24 & 386.24 & 375.81 & 370.89 & 379.32 & 386.28 & 375.28 \\
\hline T12 FYM only@10 t/ha & 389.26 & 399.16 & 413.72 & 393.97 & 395.24 & 401.18 & 410.12 & 398.12 \\
\hline SEm \pm & 13.04 & 13.69 & 14.97 & 12.21 & 13.57 & 14.31 & 10.85 & 12.58 \\
\hline CD@0.05 & 38.26 & 40.17 & 43.92 & 35.82 & 39.34 & 41.97 & 31.84 & 36.92 \\
\hline CV $(\%)$ & 8.65 & 8.13 & 7.93 & 7.81 & 7.13 & 8.69 & 7.74 & 8.24 \\
\hline
\end{tabular}


Lowest urease activity 21.34, 26.39, 28.43, 24.16 and $23.19,28.08,32.79,25.73 \mathrm{mg}$ $\mathrm{NH}_{4}{ }^{+}$released $\mathrm{g}^{-1}$ soil $\mathrm{h}^{-1}$ at initial, tillering, panicle initiation and at harvest was recorded by $\mathrm{T}_{1}$ (control).

At initial stage the highest urease activity (42.34, 43.69, 46.82 and $45.84 \mu \mathrm{g}$ of $\mathrm{NH} 4+$ released $\mathrm{g}^{-1}$ soil $\mathrm{h}^{-1}$, kharif, rabi 2016-17 and kharif, rabi 2017-18, respectively) was recorded in the treatment which received 100 $\%$ NPK + FYM @ $5 \mathrm{t} \mathrm{ha}^{-1}\left(\mathrm{~T}_{7}\right)$ and it was significantly superior over other treatments but however it was on par with $\mathrm{T}_{9}$ and $\mathrm{T}_{10}$.

At tillering stage, in both Kharif and Rabi seasons, among the all treatments the highest urease activity was observed in treatment $\left(\mathrm{T}_{7}\right)$ which was significantly superior over other treatments but however it was on par with treatment $\mathrm{T}_{10}$. The lowest urease activity observed in treatment $\mathrm{T}_{1}$ (control) in 2016-17 and 2017-18 in both the seasons.

At panicle initiation satge, the urease activity was ranged from 28.43 to 52.19 in kharif, $2016 ; 32.79$ to 55.49 in kharif $2017 ; 31.27$ to 58.94 in rabi, 2017 and 32.79 to 55.49 in rabi, 2018. During the first year kharif and rabi the highest urease activity was observed with treatment $T_{7}$ it was significantly superior over other treatments. In second year the treatment $\mathrm{T}_{7}$ was on par with $\mathrm{T}_{10}$ and significantly superior over remaining treatments and lowest urease activity was observed in control $\left(\mathrm{T}_{1}\right)$.

At harvesting stage, during kharif 2016 \& 17 the highest urease activity was observed with treatment $\mathrm{T}_{7}\left(100 \% \mathrm{NPK}+\mathrm{FYM} @ 5 \mathrm{t} \mathrm{ha}^{-1}+\right.$ $\mathrm{ZnSO}_{4}$ ) it was significantly superior over all other treatments. During rabi (2017 \&2018) the highest urease activity was observed in the treatment $\mathrm{T}_{7}$ over other treatments but it was on par with $\mathrm{T}_{10}$. Lowest urease activity was observed in control $\left(\mathrm{T}_{1}\right)$.
Among the inorganic treatments, the highest urease activity was observed in treatment $\mathrm{T}_{6}$ $\left(100 \%\right.$ RDF \%+ $\left.\mathrm{ZnSO}_{4}\right)$ and it was significantly superior over other treatments but however it was on par with treatment $\mathrm{T}_{2}$ (100\% NPK). Similar results were observed at initial, tillering, panicle initiation and harvest during both the years of study in kharif and rabi seasons.

The higher enzyme activity with $100 \%$ NPK+FYM and FYM treatments might be ascribed to the fact that the organic matter added to low land rice soil (with water more than saturation capacity) enhances microbial fermentation of the organic compounds producing compounds which are subjected to reduction and oxidation. A number of fermentation products like ethanol, acetate, lactate act as rich energy sources for proliferating microorganisms and the microorganisms release these enzymes into the soil for the reactions necessary to release energy.

The treatment with $100 \%$ recommended dose of NPK through chemical fertilizers recorded lower enzyme activities than the INM treatments which was attributed to lack of sufficient substrate i.e. organic carbon which acts as an energy source and food for proliferating the microbial population (Nagendra, 2015).

\section{Dehydrogenase activity}

The dehydrogenase activity gradually increased with the age of the crop, attained highest activity at panicle initiation stage and gradually decreased to harvest.

The higher dehydrogenase activity at panicle initiation stage was likely due to high $\mathrm{C}$ input in the soil in the form of root mass that enhanced microbial activity (Maurya et al., 2011). 
At initial stage, the highest DHA (416.31, $425.21 \mu \mathrm{g} \mathrm{TPF} \mathrm{g}{ }^{-1}$ day $^{-1}$ ) in kharif 2016 and 17 and (420.16, 427.69 $\mu \mathrm{g}$ TPF $\mathrm{g}^{-1}$ day $^{-1}$ ) in rabi 2017 and 18 was recorded in the treatment $\mathrm{T}_{7}$ and it was significantly superior over other treatments but it was on par with treatment $\mathrm{T}_{12}$ (FYM @ 10t/ha) and lowest dehydrogenase activity was observed in control $\left(\mathrm{T}_{1}\right)$ (Table 9 and 10).

At tillering satge, the dehydrogenase activity ranged from 274.89 to 424.29 in Kharif, 2016; 265.19 to 434.91in Kharif, 2017; 265.19 to 429.19 in Rabi, 2017 and 270.13 to $435.62 \mu \mathrm{g}$ TPF g ${ }^{-1}$ day $^{-1}$ in Rabi, 2018. The highest dehydrogenase activity was observed with treatment $T_{7}$ and it was significantly superior over other treatments but however it was on par with treatment $T_{10}, T_{11}$ and $T_{12}$ and lowest dehydrogenase activity was observed in control $\left(\mathrm{T}_{1}\right)$ in both the seasons and both the years of study.

At panicle initiation stage $\&$ harvest stage, the highest dehydrogenase activity in both the years of study in kharif and rabi was observed in $\mathrm{T}_{7}\left(100 \%\right.$ RD of NPK+FYM @ $10 \mathrm{t} \mathrm{ha}^{-1}+$ $\mathrm{ZnSO}_{4}$ ) and it was significantly superior over other treatments but however it was on par with $\mathrm{T}_{10}, \mathrm{~T}_{11}$ and $\mathrm{T}_{12}$ and the lowest dehydrogenase activity was observed in control $\left(\mathrm{T}_{1}\right)$ and it was on par with $\mathrm{T}_{8}(50 \%$ NPK).

Addition of organic sources might have created environment conducive for formation of humic acid, stimulated the activity of soil micro-organisms resulting in an increase in DHA of the soil (Bajpai et al., 2006).

At all the stages of crop growth during kharif, rabi 2016-17 and 2017-18 among the inorganic treatments, the treatment $\mathrm{T}_{6}(100 \%$ $\mathrm{RDF} \%+\mathrm{ZnSO}_{4}$ ) recorded the highest dehydrogenase activity and it was significantly superior over other inorganic treatments but however it was on par with treatment $\mathrm{T}_{2}(100 \% \mathrm{NPK})$.

Significant increase in enzyme activity at panicle initiation stage due to addition of organics might be due to increased bacterial population (Pauscal et al., 1998).

Highest enzyme activity in the soils applied with FYM could be attributed to the fact that it was more decomposed and was used as readily available source of carbon and energy for proliferation of microorganisms.

The increase in dehydrogenase activity in submerged soils was attributed to the increase in population of anaerobic microorganisms. There was a shift in soil microflora from predominantly aerobic to facultative and obligatory anaerobic ones once the soil is flooded. This shift from aerobic to anaerobic microorganisms was found to cause an increase in dehydrogenase activity (Lee et al., 2004).

\section{References}

Bajpai, R. K., Chitale, S., Upadhyay, S.K and Urkurkar, J.S. 2006. Long-term studies on soil physico-chemical properties and productivity of rice - wheat system as influenced by integrated nutrient management in Inceptisols of Chhattisgarh. Journal of the Indian Society of Soil Science. 54(1): 24-29.

Chakravarthi, S.N and Kar, A. K. 1970. Solubility of phosphorus in waterlogged soils. Journal of the Indian Society of Soil Science. 18: 249-257.

Kumar, V and Singh, A.P. 2010. Long-term effect of green manuring and farm yard manure on yield and soil fertility status in rice-wheat cropping system. Journal of the Indian Society of Soil Science. 58(4): 409-412. 
Laxminarayana, K. 2006. Effect of integrated use of inorganic and organic manureson soil properties, yield and nutrient uptake of rice in Ultisols of Mizoram. Journal of the Indian Society of Soil Science. 54(1): 120-123.

Lee, J.J., Park, R.D., Kim, Y.W., Shim, J.H., Chae, D.H., Rim, Y.S and Kyoon, B. 2004. Effect of food waste compost on microbial population, soil enzyme activity and lettuce growth. Bioresource Technology. 93: 21-28.

Malewer, G.V and Hasnabade, A.R. 1995. Effect of long-term application of fertilizers and organic sources on some properties of Vertisol. Journal of Maharashtra Agricultural University. 20(2): 285-286.

Maurya, B.R., Singh. V and Dhyani, P. P. 2011. Enzymatic activities and microbial population in agric-soils of Almora District of Central Himalaya as influenced by altitudes. International Journal of Soil Science 6: 238-248.

Nagendra. V. 2015. Influence of rice production systems and nutrient management practices on rice yield and soil properties. Prof. Jayashankar Telangana state Agricultural University.
Nambiar, K. K. M and Abrol, I.P. 1989. Long term fertilizer experiment in Indian over view. Fertilizer News. 34: 11-20 \&26.

Pauscal, J.A., Hernandez, T., Garcia, C and Ayuso, M. 1998. Enzymatic activities in an arid soil amended with urban organic wastes Laboratory experiments. Bio Resource Technology. 64: 131-138.

Prakash, T.R and Badrinath Ali, M.K. 1994. Relative efficiency of different phosphorus sources as influenced by liming on the yield and uptake by rice on Oxixols. Journal of Indian Society of Soil Science. 42: 271-273.

Rathore, A.L., Chipde, S.J and Pal, A.R. 1995. Direct and residual effects of bioorganic and inorganic fertilizers in rice (Oryza sativa) - wheat (Triticum aestivum) cropping system. Indian Journal of Agronomy. 40(1): 14-19.

Tandon, H.L.S. 1987. Phosphorous Research and Agricultural Production in India, Fertilizer Development and Consultation Organization (FDCO), New Delhi.

Zeng, Lu., Sheng., Liano., Min., Chen Cheng, Li and Huang, Chang-Yong. 2005. Variation of soil microbial biomass and enzyme activities at different growth stages of rice. Rice Science. 12(4): 283288.

\section{How to cite this article:}

Goutami, N., Ch. Sujani Rao, A. Sireesha, Ch. Pulla Rao and Vijaya Gopal, A. 2018. Effect of Long-Term Use of Inorganic Fertilizers, Organic Manures and Their Combination on Soil Properties and Enzyme Activity in Rice-Rice Cropping System. Int.J.Curr.Microbiol.App.Sci. 7(09): 469-486. doi: https://doi.org/10.20546/ijcmas.2018.709.057 